The Astrophysical Journal, 669:810-820, 2007 November 10

(C) 2007. The American Astronomical Society. All rights reserved. Printed in U.S.A.

\title{
PAH EMISSION FROM ULTRALUMINOUS INFRARED GALAXIES ${ }^{1}$
}

\author{
V. Desai, ${ }^{2}$ L. Armus,${ }^{3}$ H. W. W. Spoon, ${ }^{4}$ V. Charmandaris, ${ }^{5,6}$ J. Bernard-Salas ${ }^{4}$ B. R. Brande, ${ }^{7}$ \\ D. Farrah, ${ }^{4}$ B. T. Solfer, ${ }^{2,3}$ H. I. Teplitz ${ }^{3}$ P. M. Ogle, ${ }^{3}$ D. Devost, ${ }^{4}$ \\ S. J. U. Higdon, ${ }^{8}$ J. A. Marshall, ${ }^{4}$ and J. R. HoucK ${ }^{4}$ \\ Received 2007 January 7; accepted 2007 July 17
}

\begin{abstract}
We explore the relationships between the polycyclic aromatic hydrocarbon (PAH) feature strengths, mid-infrared continuum luminosities, far-infrared spectral slopes, optical spectroscopic classifications, and silicate optical depths in a sample of 107 ULIRGs observed with the Infrared Spectrograph on the Spitzer Space Telescope. The detected $6.2 \mu \mathrm{m}$ PAH equivalent widths (EWs) in the sample span more than 2 orders of magnitude $(\sim 0.006-0.8 \mu \mathrm{m})$, and ULIRGs with $\mathrm{H}$ II-like optical spectra or steep far-infrared spectral slopes $\left(S_{25} / S_{60}<0.2\right)$ typically have $6.2 \mu \mathrm{m}$ PAH EWs that are half that of lower luminosity starbursts. A significant fraction $(\sim 40 \%-60 \%)$ of $\mathrm{H}$ II-like, LINER-like, and cold ULIRGs have very weak PAH EWs. Many of these ULIRGs also have large $\left(\tau_{9.7}>2.3\right)$ silicate optical depths. The farinfrared spectral slope is strongly correlated with PAH EW, but not with silicate optical depth. In addition, the PAH EW decreases with increasing rest-frame $24 \mu \mathrm{m}$ luminosity. We argue that this trend results primarily from dilution of the PAH EW by continuum emission from dust heated by a compact central source, probably an AGN. High-luminosity, highredshift sources studied with Spitzer appear to have a much larger range in PAH EW than seen in local ULIRGs, which is consistent with extremely luminous starburst systems being absent at low redshift, but present at early epochs.
\end{abstract}

Subject headings: galaxies: active — galaxies: starburst — infrared: galaxies

\section{INTRODUCTION}

Ultraluminous infrared galaxies (ULIRGs) have bolometric luminosities comparable to quasars $\left(L_{\text {bol }} \gtrsim 10^{12} L_{\odot}\right)$, but emit nearly all of this energy at mid- and far-infrared wavelengths. Although ULIRGs are rare in the local universe and comprise only a few percent of infrared-bright galaxies (Soifer et al. 1987; Sanders et al. 2003), they account for a rapidly increasing fraction of all star formation activity at high redshift, and they may dominate the far-infrared background at $z>2$ (Franceschini et al. 2001; Le Floc'h et al. 2005; Pérez-González et al. 2005). It has been suggested that they play a role in the formation of both quasars and elliptical galaxies (Kormendy \& Sanders 1992; Sanders \& Mirabel 1996; Scott et al. 2002).

To understand the roles that ULIRGs play in the formation of massive galaxies, the global star formation history of the universe, and the creation of the cosmic infrared background, it is essential to determine the extent to which active galactic nuclei (AGNs) and star formation contribute to their enormous luminosities. Several studies have used optical (de Grijp et al. 1985; Osterbrock \& De Robertis 1985; Armus et al. 1987, 1989; Veilleux et al. 1995, 1999a; Kim et al. 1998) and near-infrared (Goldader et al. 1995; Veilleux et al. 1997, 1999b; Murphy et al. 1999, 2001; Burston et al. 2001;

\footnotetext{
1 Based on observations obtained with the Spitzer Space Telescope, which is operated by the Jet Propulsion Laboratory, California Institute of Technology, under NASA contract 1407.

2 Division of Physics, Mathematics, and Astronomy, California Institute of Technology, Pasadena, CA 91125.

3 Spitzer Science Center, MS 220-6, Caltech, Pasadena, CA 91125.

4 Astronomy Department, Cornell University, Ithaca, NY 14853.

5 University of Crete, Department of Physics, P.O. Box 2208 GR-71003, Heraklion, Greece.

6 IESL/Foundation for Research and Technology-Hellas, GR-71110, Heraklion, Greece and Chercheur Associé, Observatoire de Paris, F-75014, Paris, France.

7 Leiden Observatory, Leiden University, P.O. Box 9513, 2300 RA Leiden, Netherlands.

8 Department of Physics, Georgia Southern University, Statesboro, GA 30460.
}

Davies et al. 2003; Dannerbauer et al. 2005) spectroscopy to determine the main source of ionization of the line-emitting gas in local ULIRGs. These studies show that while the majority of ULIRGs have optical and near-infrared spectra similar to those of starburst galaxies, the fraction of ULIRGs displaying the spectroscopic signatures of an AGN, either broad permitted lines or high-ionization narrow lines, increases with infrared luminosity. In addition, evidence for AGN activity is more commonly found among ULIRGs with flatter far-infrared spectral slopes (those classified as "warm" with $S_{25} / S_{60} \geq 0.2$ as measured with $I R A S$ ) than among ULIRGs with steeper far-infrared spectral slopes (those classified as "cold" with $S_{25} / S_{60}<0.2$ ).

A principle uncertainty of these optical and near-infrared studies is the extent to which the physical conditions of the visible gas are linked to those in the deeply embedded nucleus, where the bulk of the energy is generated (Soifer et al. 2000). Emission at longer wavelengths can penetrate more heavily obscured regions close to the nuclear power source. Therefore, diagnostics derived from mid-infrared spectroscopy are potentially a better probe of the dominant energy source in the nuclear regions. The mid-infrared spectra of ULIRGs are comprised of continuum emission from heated dust grains; broad emission features associated with polycyclic aromatic hydrocarbons (PAHs), the most prominent of which appear at $6.2,7.7,8.6,11.3$, and $12.7 \mu \mathrm{m}$; absorption by amorphous silicates centered at 9.7 and $18 \mu \mathrm{m}$; and atomic finestructure lines of $\mathrm{Ne}, \mathrm{O}, \mathrm{Si}$, and $\mathrm{S}$ covering a large range in ionization potential. Diagnostic diagrams using combinations of fine-structure line ratios, mid-infrared spectral slope, and $\mathrm{PAH}$ feature strengths have been used to classify bright ULIRGs, based on the expectations that ULIRGs with central AGNs produce highionization lines, have flatter spectral slopes, and display lower PAH equivalent widths than those without (e.g., Genzel et al. 1998; Lutz et al. 1998; Rigopoulou et al. 1999; Laurent et al. 2000; Tran et al. 2001; Sturm et al. 2002; Armus et al. 2004, 2006, 2007; Farrah et al. 2007). For sources at high-redshift $(z \geq 1)$, however, it is generally not possible to place useful limits on the 
high-ionization fine-structure lines. The PAH emission, together with the infrared spectral slope, are often the only mid-infrared diagnostics available for studying high-redshift ULIRGs.

The first attempts to classify the energy generation mechanism in ULIRGs using mid-infrared PAH features made use of data from the Infrared Space Observatory (ISO; Lutz et al. 1998, 1999; Rigopoulou et al. 1999; Tran et al. 2001). For these studies, ULIRGs were classified based on the strengths of their $7.7 \mu \mathrm{m}$ PAH line-to-continuum ratios $(L / C$, defined as the ratio of the peak height of the $7.7 \mu \mathrm{m}$ PAH feature to the level of the underlying $7.7 \mu \mathrm{m}$ continuum). The PAH-based classifications showed that most ( $\sim 80 \%$ ) ULIRGs have strong PAH emission indicative of star formation as the dominant power source. However, the highest luminosity sources tend to have weak (or undetected) PAH emission, interpreted as a sign of increased AGN activity. Recently, Imanishi et al. (2007) analyzed the mid-infrared spectra of 48 nearby ULIRGs observed with the Infrared Spectrograph (IRS; ${ }^{9}$ Houck et al. 2004) on board the Spitzer Space Telescope. These authors suggest that $30 \%-50 \%$ of these ULIRGs, all of which have $\mathrm{H}$ II-like or LINER-like optical classifications, harbor buried AGNs.

In this paper we present the mid-infrared spectroscopic properties of a large sample of 107 ULIRGs that comprise the IRS GTO team ULIRG survey. The sample includes a large fraction ( $\sim 40 \%$ ) of warm sources, which tend to have higher infrared luminosities than cold ULIRGs. The warm ULIRGs are a critical population to study, since it has been suggested that they mark the transition between starburst-dominated ULIRGs and QSOs (e.g., Sanders et al. 1988b). Because of their high luminosities, they provide a much-needed baseline over which to extrapolate to the extremely high luminosities $\left(L_{\mathrm{IR}}>10^{13} L_{\odot}\right)$ seen at higher redshift.

The sensitivity of the IRS offers two distinct advantages over previous studies with ISO. First, we can measure the PAH strengths with respect to the dust continuum (the equivalent width, hereafter EW ) over nearly 2 orders of magnitude, allowing us to quantify the PAH emission in many ULIRGs that previously only had upper limits. This is critical for fitting trends with luminosity and spectral slope, as many ULIRGs have much weaker PAH emission features than are typically found in low-luminosity starburst galaxies (see $\S 3.1$ ). Second, the Short-Low and Long-Low IRS modules $(5-38 \mu \mathrm{m})$ offer greatly increased wavelength coverage compared to ISOPHOT-S, allowing us to accurately fit the silicate absorption (at 9.7 and $18 \mu \mathrm{m}$ ) and multiple PAH features in all the ULIRGs. Here, we have chosen to fit the $9.7 \mu \mathrm{m}$ silicate absorption feature since it is the strongest and the 6.2 and $11.3 \mu \mathrm{m}$ PAH features because they are both well isolated and easily measured. Previously the $7.7 \mu \mathrm{m}$ feature was often used, which is not ideal because absorption at both shorter and longer wavelengths can mimic a peak at $7.7 \mu \mathrm{m}$ (Spoon et al. 2002) and its integrated flux can be difficult to measure due to blending with the adjacent $8.6 \mu \mathrm{m}$ feature. By correlating the PAH emission with the silicate absorption, it is possible to gain insight into the distribution of the heating source(s) and the grains. In the following analysis, we use $H_{0}=70 \mathrm{~km} \mathrm{~s}^{-1} \mathrm{Mpc}^{-1}, \Omega_{m}=0.3$, and $\Lambda=0.70$.

\section{OBSERVATIONS AND DATA REDUCTION}

The 107 ULIRGs presented in this paper were chosen primarily from the IRAS 1 Jy survey (Kim et al. 1998), the IRAS 2 Jy survey (Strauss et al. 1992), and the FIRST/IRAS radio-far-infrared sample

\footnotetext{
9 The IRS was a collaborative venture between Cornell University and Ball Aerospace Corporation funded by NASA through the Jet Propulsion Laboratory and the Ames Research Center.
}

of Stanford et al. (2000). The ULIRGs span a redshift range of $0.018<z<0.93$, have IRAS $60 \mu \mathrm{m}$ flux densities between $0.14<\mathrm{S}_{60} / \mathrm{Jy}<103$, and have integrated $40-500 \mu \mathrm{m}$ luminosities in the range $11.7<\log \left(L_{\mathrm{FIR}} / L_{\odot}\right)<13.13$. The sample consists of 41 warm ULIRGs and 66 cold ULIRGs, based on their rest-frame far-infrared flux densities at 25 and $60 \mu \mathrm{m}$. Many of our highest luminosity sources were taken from the Stanford et al. (2000) FIRST/IRAS survey. While radio-bright AGNs may preferentially populate the highest luminosities of the Stanford et al. survey, the selected ULIRGs have radio to far-infrared flux density ratios consistent with infrared-selected starburst galaxies of lower luminosity. The sample is specifically designed to include a larger fraction of warm and infrared luminous sources than would be found in a pure flux or volume-limited sample, for the express purpose of allowing a careful study of the mid-infrared spectral properties as a function of infrared color and luminosity.

All ULIRGs were observed in Staring Mode with both subslits (orders) of each of the Short-Low (SL) and Long-Low (LL) modules of the IRS. Each target was acquired by performing a highaccuracy IRS peak-up on the target itself, or by peaking up on a nearby 2MASS star and offsetting to the target. Each galaxy was observed at two nod positions within each of the IRS subslits (SL1, SL2, LL1, LL2). The resulting spectra have a spectral resolution of $R \sim 80$ over the $5-38 \mu \mathrm{m}$ wavelength range.

All spectra were reduced using the S14 IRS pipeline at the Spitzer Science Center. This reduction includes ramp fitting, dark sky subtraction, droop correction, linearity correction, and wavelength and flux calibration. The flux calibration sources were HD 173511 for the SL and LL2 modules and KsiDra for the LL1 module. For a given module, order, and nod position, the background in the two-dimensional spectrum was removed by subtracting the combined two-dimensional data taken with the same module, but adjacent order. One-dimensional spectra were extracted from the background-subtracted two-dimensional spectra using the SMART data reduction package (Higdon et al. 2004). The adopted extraction apertures are four pixels at the blue end of each order and expand linearly with wavelength.

The 6.2 and $11.3 \mu \mathrm{m}$ PAH features were measured by integrating the flux above a spline-interpolated continuum. For those sources with strong $6 \mu \mathrm{m}$ water ice absorption, the apparent $6.1 \mu \mathrm{m}$ PAH EW has been corrected by using an inferred $6.2 \mu \mathrm{m}$ continuum defined by a spline interpolation between $5-26 \mu \mathrm{m}$, using pivot points at 5.2, 5.6, 7.8 (in the case of very weak PAH emission), 14, and $26 \mu \mathrm{m}$. This correction results in a lower $6.2 \mu \mathrm{m}$ PAH EW, and is correct under the assumption that the PAH emission is unaffected by the bulk of the ice absorption. A full description of the ice-fitting procedure is given in Spoon et al. (2007), where the measured $6.1 \mu \mathrm{m}$ EWs for many of these ULIRGs are first presented (see $\S 3.2$ and Fig. 2 for a description of the correction and Fig. 1 for an indication of the size of the correction). The PAH emission at $11.3 \mu \mathrm{m}$ is affected by the broad $9.7 \mu \mathrm{m}$ silicate absorption feature, which can be deep in ULIRGs. However, lacking a strong understanding of how the obscuring dust is distributed, we have not attempted to correct for it. Of the 107 ULIRGs analyzed in this paper, the $6.2 \mu \mathrm{m}$ PAH feature is detected for all but 11 sources, and the $11.3 \mu \mathrm{m}$ PAH feature is detected for all but 12 sources.

In the following, we investigate the variation of the $\mathrm{PAH}$ features with rest-frame continuum fluxes at 5.5, 24, 25, and $60 \mu \mathrm{m}$. The $5.5 \mu \mathrm{m}$ flux is taken to be the average continuum between 5.3 and $5.8 \mu \mathrm{m}$. The $24 \mu \mathrm{m}$ flux was calculated by convolving the IRS spectrum with the MIPS $24 \mu \mathrm{m}$ bandpass. In the 10 cases where the IRS spectrum did not extend over the full wavelength range of the wide IRS bandpass due to trimming 

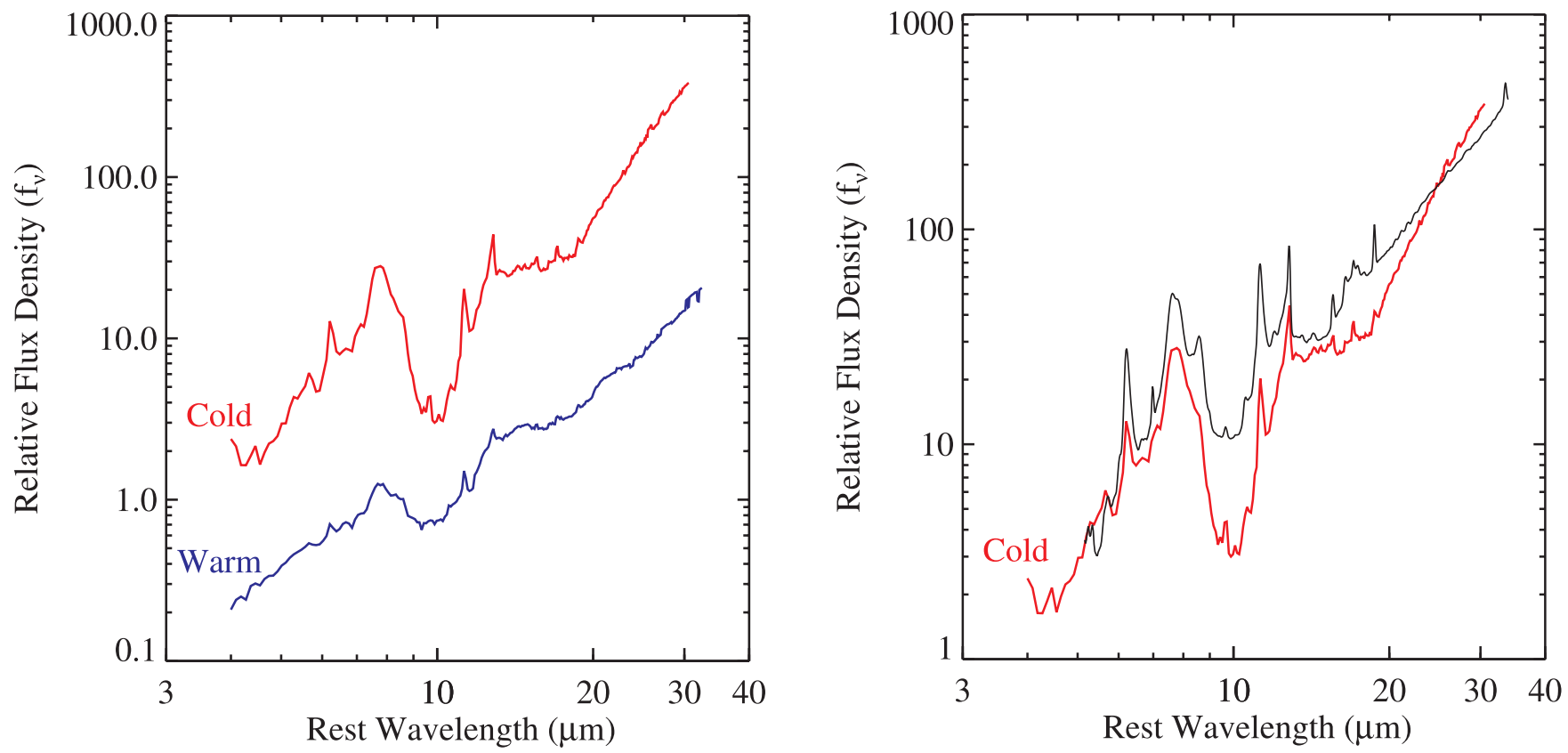

FIG. 1.-Left: Median IRS spectra of ULIRGs classified as cold (red) and warm (blue), arbitrarily normalized for display purposes. Right: Median spectrum of cold ULIRGs (red) compared to the average starburst spectrum from Brandl et al. (2006) (black), both normalized at $24 \mu \mathrm{m}$.

of pipeline artifacts (e.g., fringes), it was extended by linearly interpolating in log-log space between the IRS spectrum and the $60 \mu \mathrm{m}$ IRAS flux density (Moshir 1990). No color-correction was made to place these filter fluxes on the MIPS absolute scale, but these corrections are small $(<5 \%)$ based on the spectral shape of the ULIRGs within the $24 \mu \mathrm{m}$ bandpass (see the MIPS Data Handbook). The $25 \mu \mathrm{m}$ rest-frame flux was estimated by taking the median of the trimmed and (when needed) interpolated IRS spectrum between 24.7 and $25.3 \mu \mathrm{m}$. The rest-frame $60 \mu \mathrm{m}$ luminosity was estimated by linearly interpolating in log-log space between the observed IRAS 60 and $100 \mu \mathrm{m}$ flux densities. For 12 ULIRGs, this interpolation likely underestimates the $60 \mu \mathrm{m}$ luminosity, either because the object is at a redshift high enough $(z>0.65)$ that the observed $100 \mu \mathrm{m}$ IRAS observation corresponds to a restframe wavelength less than $60 \mu \mathrm{m}$, or because the $100 \mu \mathrm{m}$ observation resulted in only a limit, rather than a detection.

Classifications based on optical spectra are available from the literature for 64 of the 107 ULIRGs in our sample. Of these, 15 have $\mathrm{H}$ II-like optical spectra, 22 are LINER-like, and 27 are Seyfert-like.

\section{RESULTS}

\subsection{PAH Strength and Silicate Absorption versus Spectral Slope and Optical Classification}

The median IRS spectra for warm and cold ULIRGs are shown in the left-hand panel of Figure 1. Cold ULIRGs have a median $6.2 \mu \mathrm{m}$ PAH EW that is a factor of 6 higher than the median measured for warm ULIRGs $(0.24$ vs. $0.04 \mu \mathrm{m}$; see Table 1). The right-hand panel of Figure 1 shows the average starburst spectrum from Brandl et al. (2006) plotted over the median cold ULIRG spectrum. Cold ULIRGs have a median $6.2 \mu \mathrm{m}$ PAH EW which is about half the median for lower luminosity starbursts (see also Imanishi et al. 2007). The difference in the PAH EWs in the median warm and cold ULIRG spectra reflects the strong trend between PAH EW and mid-infrared spectral type

TABLE 1

6.2 and $11.3 \mu \mathrm{m}$ PAH EWs versus Spectral Class

\begin{tabular}{|c|c|c|c|c|c|c|c|}
\hline $\begin{array}{c}\text { Spectral Class } \\
\text { (1) }\end{array}$ & $\begin{array}{l}\text { NUMBer } \\
\text { (2) }\end{array}$ & \multicolumn{2}{|c|}{ UNCORRECTED $6.2 \mu \mathrm{m}$ PAH EW } & \multicolumn{2}{|c|}{ CoRrected $6.2 \mu \mathrm{m}$ PAH EW } & \multicolumn{2}{|c|}{$11.3 \mu \mathrm{m}$ PAH EW } \\
\hline 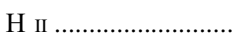 & 15 & 0.32 & $0.30 \pm 0.16$ & 0.28 & $0.27 \pm 0.18$ & 0.39 & $0.41 \pm 0.20$ \\
\hline LINER ......................... & 22 & 0.19 & $0.27 \pm 0.20$ & 0.11 & $0.17 \pm 0.14$ & 0.50 & $0.46 \pm 0.18$ \\
\hline 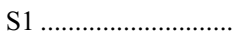 & 6 & 0.02 & $0.04 \pm 0.05$ & 0.02 & $0.04 \pm 0.05$ & 0.03 & $0.03 \pm 0.03$ \\
\hline 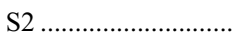 & 21 & 0.05 & $0.10 \pm 0.11$ & 0.05 & $0.08 \pm 0.07$ & 0.09 & $0.16 \pm 0.15$ \\
\hline 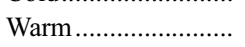 & 41 & 0.04 & $0.08 \pm 0.09$ & 0.04 & $0.06 \pm 0.08$ & 0.07 & $0.13 \pm 0.13$ \\
\hline
\end{tabular}

Notes.-Statistics on the 6.2 and $11.3 \mu \mathrm{m}$ PAH EWs of the ULIRGs in our sample, broken down by spectral class (col. [1]). Col. (2) gives the number of ULIRGs in each class. Cols. (3) and (4) show the median and average $6.2 \mu \mathrm{m}$ PAH EWs, respectively, uncorrected for ice absorption. Cols. (5) and (6) give the same statistics for the ice-corrected $6.2 \mu \mathrm{m}$ PAH EW (see Spoon et al. (2007) for details on this correction). Col. (7) shows the median and average $11.3 \mu \mathrm{m}$ PAH EWs, uncorrected for the underlying silicate absorption. All medians and averages were computed by including sources with upper limits as although they were detections. Optical classifications are from Duc et al. (1997); Veilleux et al. (1995, 1997); Cutri et al. (1994); Allen et al. (1991); Armus et al. (1989); Kleinmann et al. (1988); Sanders et al. (1988b). The far-infrared classifications of cold $\left[f_{\nu}(25 \mu \mathrm{m}) / f_{\nu}(60 \mu \mathrm{m})<0.2\right]$ and warm $\left[f_{\nu}(25 \mu \mathrm{m}) / f_{\nu}(60 \mu \mathrm{m}) \geq 0.2\right]$ are based on the rest-frame 25 and $60 \mu \mathrm{m}$ values calculated as described in $\S 2$. 


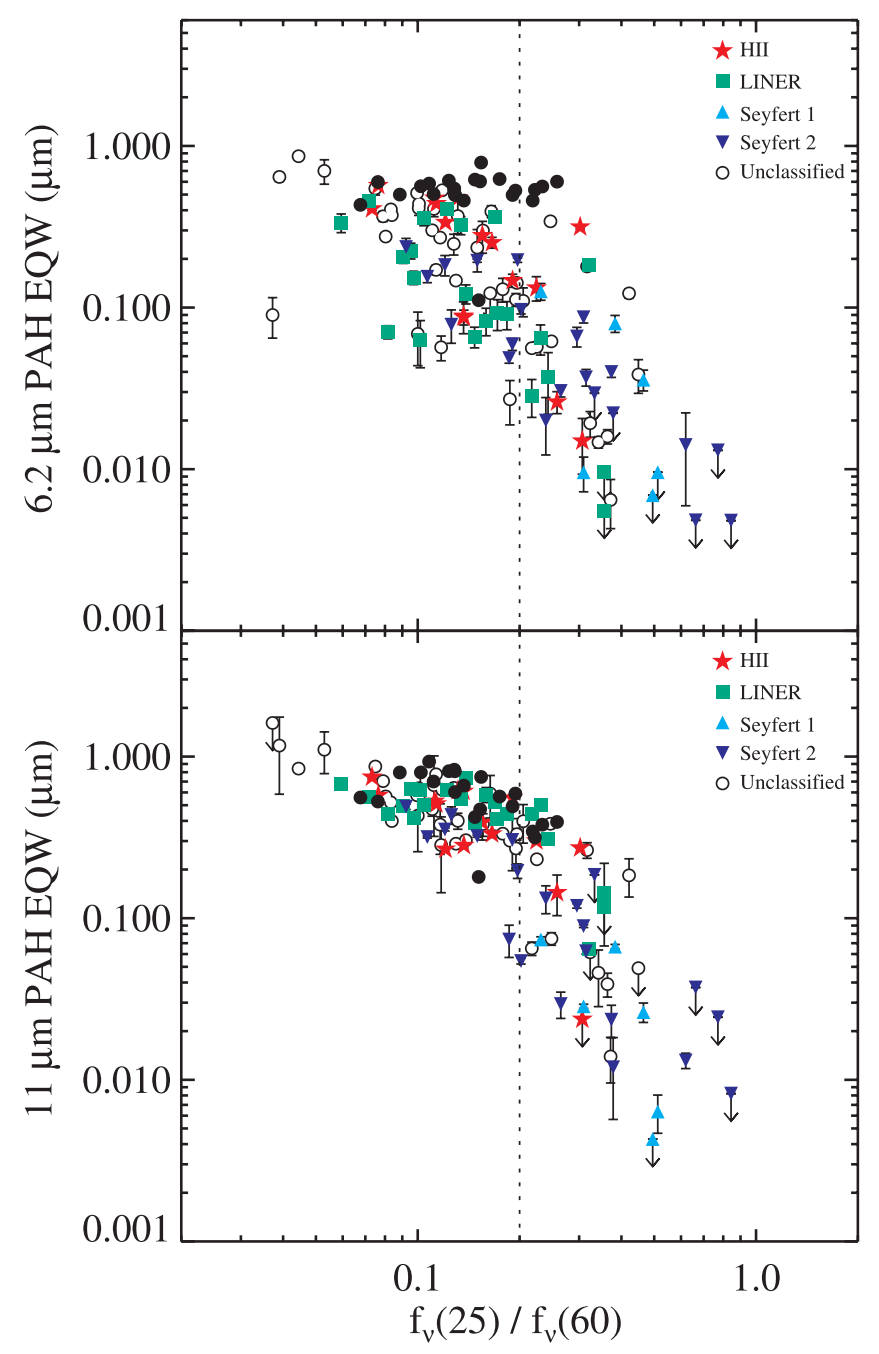

Fig. 2.-The 6.2 (top) and $11.3 \mu \mathrm{m}$ (bottom) PAH EWs vs. spectral slope. The ULIRGs are color-coded by optical spectroscopic classification: ULIRGs with $\mathrm{H}$ IIlike optical spectra are plotted as red stars, LINER-like ULIRGs are shown as green squares, ULIRGs with Seyfert 1 optical spectra are shown as light blue triangles, ULIRGs with Seyfert 2 optical spectra are shown as dark blue upside-down triangles, and sources with unknown optical spectroscopic classifications are shown as white circles. The black dots represent the starburst galaxies from Brandl et al. (2006). The dotted vertical line represents the division between warm and cold sources adopted for this paper $\left[f_{\nu}(25) / f_{\nu}(60)=0.2\right]$, where cold sources lie to the left of the line and warm sources lie to the right.

shown in Figure 2. There is a huge range in measured PAH EW among the sample ULIRGs. The full range in PAH EW for those ULIRGs with detected emission is $0.006-0.864 \mu \mathrm{m}$ for the $6.2 \mu \mathrm{m}$ feature, and $0.006-1.169 \mu \mathrm{m}$ for the $11.3 \mu \mathrm{m}$ feature. The smallest upper limits are 0.005 and $0.004 \mu \mathrm{m}$ for the 6.2 and $11.3 \mu \mathrm{m}$ features, respectively. While the median $6.2 \mu \mathrm{m}$ PAH EW among cold/H II ULIRGs is about half as large as for local starburst galaxies, the median $11.3 \mu \mathrm{m}$ PAH EW in cold/H II ULIRGs is $80 \%$ of that measured in starburst galaxies. The cold ULIRGs and starburst galaxies track each other over the limited range of $0.06<S_{25} / S_{60}<0.2$ (see Fig. 2).

Figure 1 also demonstrates that the median cold source shows stronger silicate absorption at both 9.7 and $18 \mu \mathrm{m}$ than the median warm source (1.36 vs. 0.65 for $\tau_{9.7}$, the optical depth at $9.7 \mu \mathrm{m}$ ), and the median cold ULIRG has stronger absorption than the average starburst (1.36 vs. 0.22). In Figure 3, we plot the apparent silicate optical depth as a function of far-infrared spectral

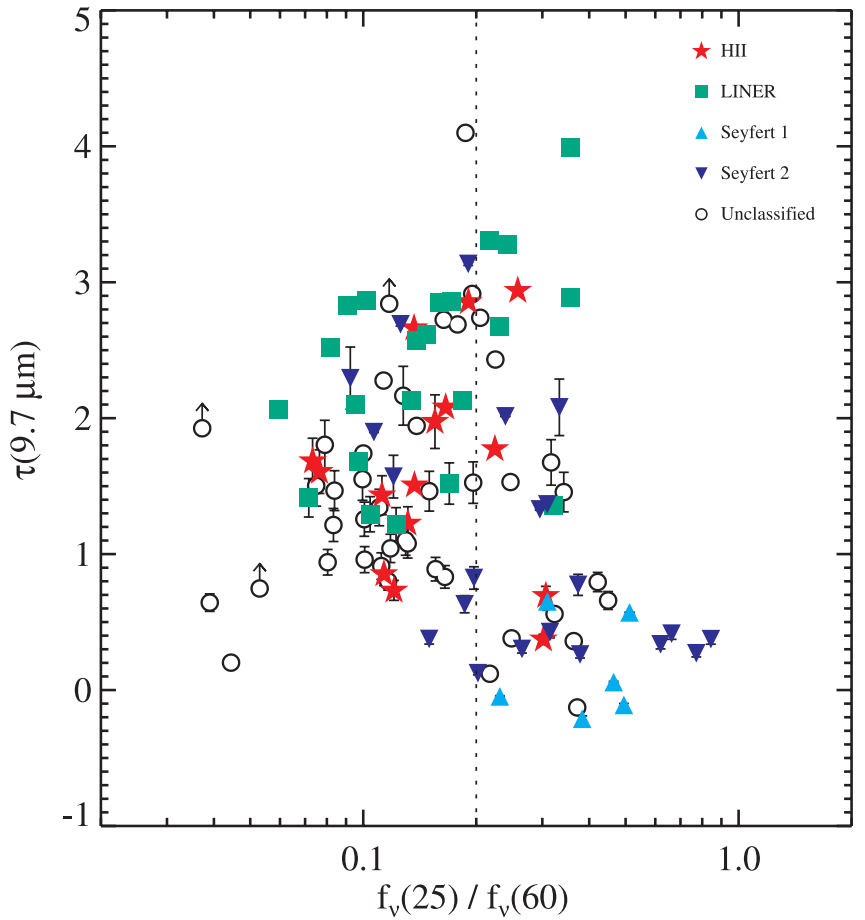

FIG. 3.-The $9.7 \mu \mathrm{m}$ silicate optical depth vs. the mid-infrared spectral slope. The ULIRGs are color-coded by optical spectroscopic classification, as in Fig. 2. The vertical dotted line is $f_{\nu}(25) / f_{\nu}(60)=0.2$, the division between warm and cold sources adopted for this paper. The spectral slope for the ULIRGs was computed from the IRS spectra as described in $\S 2$.

slope. While the ULIRGs with $\tau_{9.7}>1$ have steeper far-infrared spectra than the sources with $\tau_{9.7}<1$, we find no real correlation (see also Imanishi et al. 2007; Hao et al. 2007). In fact, many sources with very high silicate optical depths $\left(\tau_{9.7}>2\right)$ would also be classified as warm ULIRGs. All of the ULIRGs with Seyfert 1 optical spectra, and many, but not all, of the ULIRGs with Seyfert 2 optical spectra have $\tau_{9.7}<1$ and $S_{25} / S_{60}>0.2$.

The median H II, LINER, and Seyfert ULIRG IRS spectra are shown in the left-hand panel of Figure 4. The median and mean values of the 6.2 and $11.3 \mu \mathrm{m}$ PAH EWs for each of these spectral classes is given in Table 1 . As expected, the $\mathrm{H}$ II-like ULIRGs have the largest median $6.2 \mu \mathrm{m}$ PAH EW $(0.28 \mu \mathrm{m})$, while the ULIRGs classified as Seyfert galaxies have the lowest $(0.04 \mu \mathrm{m})$. The median PAH EW for infrared cold ULIRGs is comparable to the median for those classified as $\mathrm{H}$ II-like, while the median PAH EW for infrared warm sources is similar to the median for Seyfertlike ULIRGs. There are a number of ULIRGs with $\mathrm{H}_{\mathrm{II}}$-like or LINER-like spectra that have unusually low $6.2 \mu \mathrm{m}$ PAH EW ( $\lesssim 0.15 \mu \mathrm{m}$, or about $1 / 4$ that found for pure starburst galaxies). Of the 22 LINER-like (15 $\mathrm{H}$ II-like) ULIRGs in the sample, 12 (six) have $6.2 \mu \mathrm{m}$ PAH EW $\leq 0.15 \mu \mathrm{m}$. Similarly, of the 66 cold ULIRGs, 23 have a $6.2 \mu \mathrm{m}$ PAH EW $\leq 0.15 \mu \mathrm{m}$.

The right-hand panel of Figure 4 shows the median $\mathrm{H}$ II and LINER ULIRG spectra overplotted with the average starburst spectrum from Brandl et al. (2006) and the average infrared-bright LINER spectrum from Sturm et al. (2006). Although sources with $\mathrm{H}$ II-like optical spectra have the largest PAH EWs among ULIRGs, the median values are only about $50 \%$ of those measured for pure starburst galaxies, the same result as found for the cold ULIRGs. H II-like ULIRGs also have stronger silicate absorption at 9.7 and $18 \mu \mathrm{m}$ than seen in lower luminosity starbursts (1.52 vs. 0.22 for $\tau_{9.7}$ ). Compared to infrared-bright LINERs, 

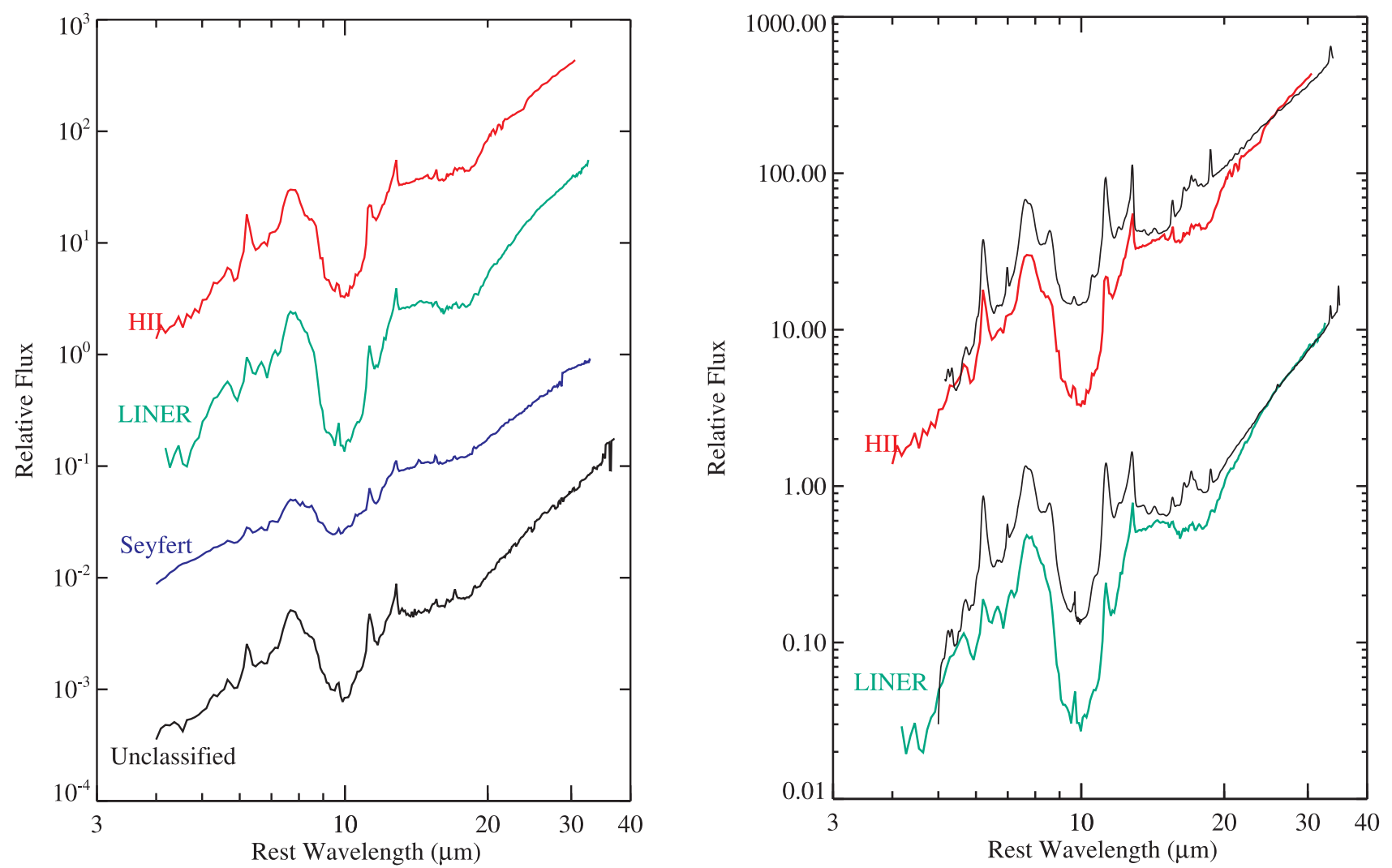

FIG. 4.-Left: Colored lines show the median IRS spectra of ULIRGs optically classified as H II-like (red), LINER-like (green), and Seyfert-like (blue). The black line shows the median spectrum of ULIRGs lacking optical classifications. The normalizations are arbitrary. Right: The median spectrum of $\mathrm{H}$ II-like ULIRGs compared to the average starburst spectrum from Brandl et al. (2006) (top red and black lines, respectively) and the median spectrum of LINER-like ULIRGs compared to the average infraredbright LINER spectrum from Sturm et al. (2006) (bottom green and black lines, respectively). For wavelengths longer than $10 \mu \mathrm{m}$, the high-resolution Sturm et al. (2006) spectrum was convolved with a Gaussian profile with a FWHM of $0.2 \mu \mathrm{m}$ to approximately match the resolution of the low-resolution ULIRG spectrum. The comparison spectra were normalized to match the $\mathrm{H}$ II- and LINER-like ULIRGs at $24 \mu \mathrm{m}$.

LINER-like ULIRGs have $~ 25 \%$ smaller PAH EWs, and stronger silicate absorption $\left(\tau_{9.7}=2.11\right.$ for the LINER-like ULIRGs, while it is only 0.84 for the IR-bright LINERs).

\subsection{PAH Equivalent Width versus Continuum Luminosity}

In Figure 5 we plot the 6.2 and $11.3 \mu \mathrm{m}$ PAH EWs versus $24 \mu \mathrm{m}$ rest-frame luminosity. This continuum wavelength was chosen because it samples the peak emission of warm $(\sim 120 \mathrm{~K})$ thermally emitting dust, it can be measured much more accurately than luminous infrared luminosity (LIR) in many cases, it is free from strong emission or absorption features, and it allows direct comparison to a variety of low-redshift samples observed with the MIPS $24 \mu \mathrm{m}$ filter and to $z \sim 2$ samples observed with the MIPS $70 \mu \mathrm{m}$ filter. For reference, we estimate that $\log _{10}\left[\nu L_{\nu}(24 \mu \mathrm{m}) / \mathrm{LIR}\right]=$ $-1.06 \pm 0.47$ for cold ULIRGs and $-0.79 \pm 0.38$ for warm ULIRGs. These estimates were made by applying the Sanders \& Mirabel (1996) prescription for converting IRAS photometry into LIR to the nearest $(z<0.2)$ ULIRGs in our sample. Figure 5 shows a trend of decreasing 6.2 and $11.3 \mu \mathrm{m}$ PAH EW with increasing rest-frame $24 \mu \mathrm{m}$ luminosity. There is significant scatter in this relation, and ULIRGs with low PAH EW span a wide range in luminosity. Nevertheless, the presence of a correlation is verified by a highly significant Spearman correlation coefficient, whether or not upper limits are included in the same way as detections in the calculation.

We have divided the ULIRG sample into six luminosity bins chosen to span the full range in $24 \mu \mathrm{m}$ luminosity [ $(0.03-8) \times$ $\left.10^{12} L_{\odot}\right]$, and to contain approximately equal numbers $(17-18)$ of ULIRGs. The median 6.2 and $11.3 \mu \mathrm{m}$ PAH EWs drop by an order of magnitude between the first and last luminosity bins (see Table 2). The median ULIRG spectrum in each $24 \mu \mathrm{m}$ luminosity class is shown in Figure 6. The median spectra were constructed using all sources in a given luminosity bin, even those for which only an upper limit on the PAH EW could be measured. Moving from lowest to highest $24 \mu \mathrm{m}$ luminosity (Fig. 6, top to bottom), the PAH features diminish and the spectrum flattens. While the median spectrum in the highest luminosity bin has a noticeably smaller silicate absorption than the lower luminosity median spectra, there is a large range in the apparent silicate optical depth among the most luminous sources. This is consistent with the results of Spoon et al. (2007), who find a dichotomy in silicate optical depths among sources with small $6.2 \mu \mathrm{m}$ PAH EW (see also $\S 3.3)$.

\subsection{Silicate Absorption versus PAH Equivalent Width}

Figure 7 shows the silicate optical depth versus $6.2 \mu \mathrm{m}$ PAH EW for the ULIRGs in our sample. Such a plot was first presented by Spoon et al. (2007), who discussed the presence of two discrete tracks, or branches. Figure 7 shows that the horizontal branch is populated almost exclusively by infrared warm sources, while the diagonal branch is populated by about half of the warm sources and virtually all of the cold sources. Examining the ULIRGs for which we have optical spectroscopic classifications, the diagonal branch harbors about half of the Seyfert-like ULIRGs, and nearly all of the $\mathrm{H}$ II-like and LINER-like ULIRGs. The horizontal branch is populated mainly by ULIRGs showing Seyfert-like spectra, 


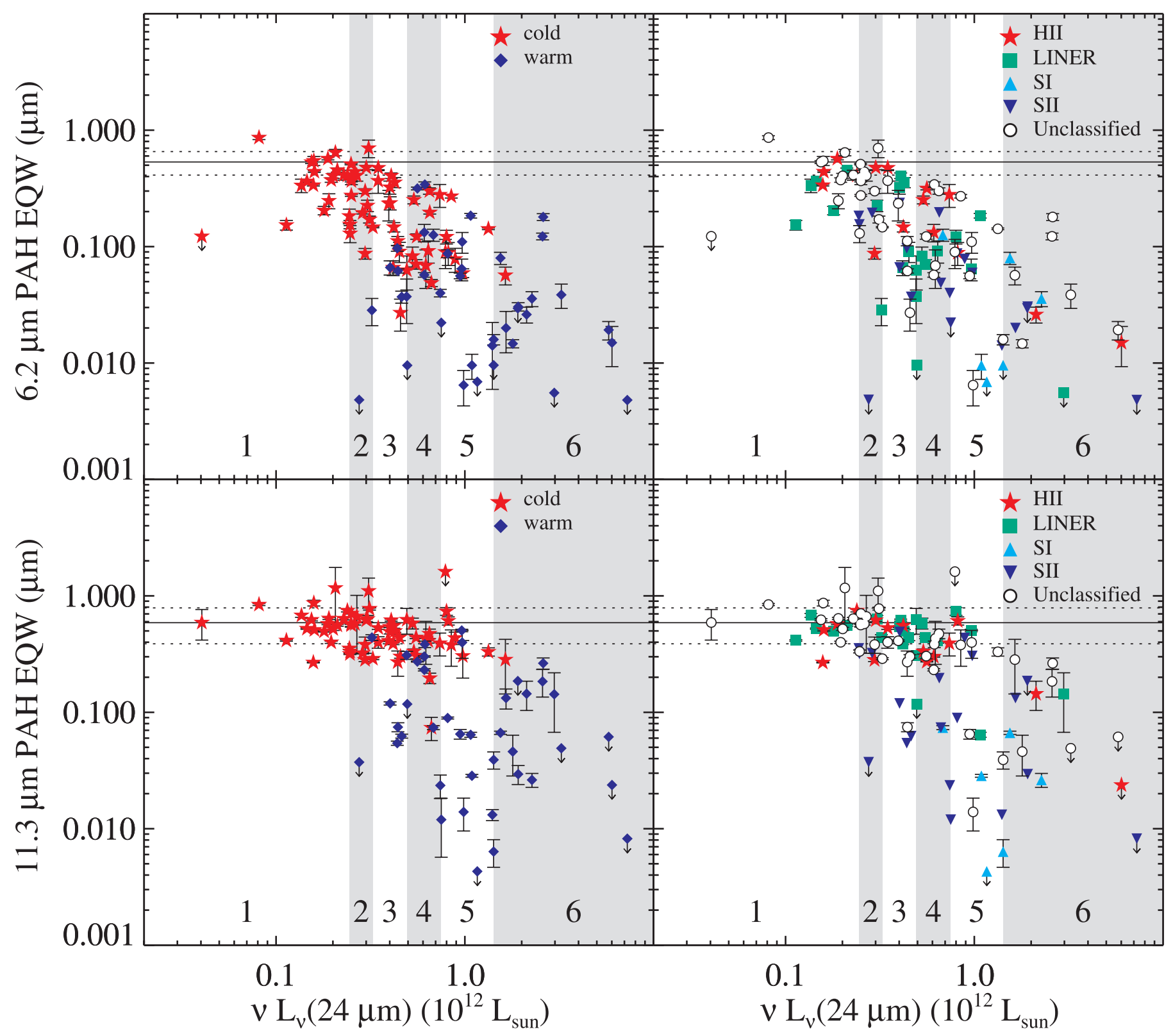

Fig. 5. - The 6.2 (top row) and $11.3 \mu \mathrm{m}$ (bottom row) PAH EWs vs. $24 \mu \mathrm{m}$ rest-frame luminosity. The ULIRG sample is color-coded by warm $\left[f_{\nu}(25 \mu \mathrm{m}) /\right.$ $\left.\left.f_{\nu}(60 \mu \mathrm{m}) \geq 0.2\right]\right)$ and cold $\left[f_{\nu}(25 \mu \mathrm{m}) / f_{\nu}(60 \mu \mathrm{m})<0.2\right]$ mid-infrared spectral slope $(l e f t)$ and by optical spectroscopic classification as in Fig. $3(r i g h t)$. The average PAH EWs of starburst galaxies from Brandl et al. (2006) are indicated by solid horizontal lines and the $\pm 1 \sigma$ dispersions in these averages are shown by dotted horizontal lines. Upper limits $(3 \sigma)$ to the PAH EWs are indicated by downward pointing arrows. The six luminosity classes (1-6; see text and Table 2$)$ are labeled on the bottom of each panel and are alternately shaded for clarity.

including all of the Type I sources. ULIRGs with Seyfert Type 2 optical spectra are found on both branches, spanning (nearly) the full range in silicate optical depth. Most of the ULIRGs without optical spectroscopic classifications lie on the diagonal branch. As noted in $\S 3.1$, a significant fraction of the $\mathrm{H}$ II and LINER-like ULIRGs have very low $6.2 \mu \mathrm{m}$ PAH EWs $(\leq 0.15 \mu \mathrm{m})$. Of these, almost all of the LINERs (11/12) and half (3/6) of the $\mathrm{H}$ II-like ULIRGs have $\tau_{9.7} \geq 2.5$, implying $A_{V} \geq 45$ mag.

\section{DISCUSSION}

The median $6.2 \mu \mathrm{m}$ PAH EWs of cold (or H II-like) ULIRGs is about $50 \%$ of that seen in lower luminosity starbursts. Warm ULIRGs have $6.2 \mu \mathrm{m}$ PAH EWs that are up to 2 orders of magnitude smaller than those found in starbursts. There are several possible explanations for the extremely large range in PAH EW and the overall low PAH EW in ULIRGs compared to starbursts. Some ULIRGs may host a central AGN, and the associated soft $\mathrm{X}$-ray and ultraviolet radiation may destroy PAH molecules (e.g., Aitken \& Roche 1985; Voit 1992), or thermalize the emission. The AGN may also heat dust to significant temperatures $(>400 \mathrm{~K})$, resulting in excess continuum emission that would lower the measured 6.2 $\mu \mathrm{m}$ PAH EW, in effect diluting the starburst-driven PAH emission with respect to the AGN-powered, hot dust continuum. For either of these explanations, $(55 \pm 13) \%$ of the LINER-like ULIRGs and $(40 \pm 15) \%$ of the $\mathrm{H}$ II-like ULIRGs in our sample would harbor buried AGN. In comparison, Lutz et al. (1999) find the percentage of LINER-like ULIRGs classified by ISO as AGNs to be $(17 \pm 15) \%$ and the percentage of $\mathrm{H}$ II-like ULIRGs classified by ISO as AGNs to be $(20 \pm 12) \%$. We are thus finding a much higher fraction of LINERs and $\mathrm{H}$ II-like ULIRGs with weak 
TABLE 2

6.2 AND $11.3 \mu \mathrm{m}$ PAH EWs versus $24 \mu \mathrm{m}$ LuMinosity

\begin{tabular}{|c|c|c|c|c|c|c|c|}
\hline \multicolumn{2}{|c|}{$24 \mu \mathrm{m}$} & \multicolumn{2}{|c|}{ UNCORRECTED $6.2 \mu \mathrm{m}$ PAH EW } & \multicolumn{2}{|c|}{ Corrected $6.2 \mu \mathrm{m}$ PAH EW } & \multicolumn{2}{|c|}{$11.3 \mu \mathrm{m}$ PAH EW } \\
\hline Class & $\begin{array}{l}\text { Luminosity } \\
\left(10^{12} L_{\odot}\right)\end{array}$ & $\begin{array}{c}\text { Median } \\
(\mu \mathrm{m})\end{array}$ & $\begin{array}{c}\text { Average } \\
(\mu \mathrm{m})\end{array}$ & $\begin{array}{c}\text { Median } \\
(\mu \mathrm{m})\end{array}$ & $\begin{array}{l}\text { Average } \\
(\mu \mathrm{m})\end{array}$ & $\begin{array}{c}\text { Median } \\
(\mu \mathrm{m})\end{array}$ & $\begin{array}{c}\text { Average } \\
(\mu \mathrm{m})\end{array}$ \\
\hline 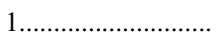 & $0.03-0.20$ & 0.43 & $0.47 \pm 0.16$ & 0.41 & $0.41 \pm 0.18$ & 0.58 & $0.61 \pm 0.20$ \\
\hline $2 \ldots \ldots \ldots \ldots \ldots \ldots \ldots$ & $0.20-0.30$ & 0.33 & $0.34 \pm 0.18$ & 0.29 & $0.30 \pm 0.17$ & 0.56 & $0.52 \pm 0.24$ \\
\hline $3 \ldots \ldots \ldots \ldots \ldots \ldots \ldots \ldots$ & $0.30-0.44$ & 0.16 & $0.23 \pm 0.17$ & 0.10 & $0.17 \pm 0.13$ & 0.44 & $0.39 \pm 0.18$ \\
\hline 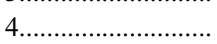 & $0.44-0.63$ & 0.14 & $0.17 \pm 0.11$ & 0.12 & $0.15 \pm 0.11$ & 0.31 & $0.30 \pm 0.15$ \\
\hline 5 & $0.63-1.15$ & 0.10 & $0.12 \pm 0.10$ & 0.06 & $0.07 \pm 0.05$ & 0.19 & $0.31 \pm 0.40$ \\
\hline 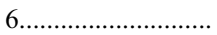 & $1.15-8.00$ & 0.03 & $0.04 \pm 0.05$ & 0.02 & $0.04 \pm 0.05$ & 0.05 & $0.08 \pm 0.08$ \\
\hline
\end{tabular}

Notes.-Same as Table 1, except the ULIRG sample is broken down into luminosity bins, rather than spectral class. Each bin contains 17-18 ULIRGs.

PAH emission, although, given the small sample sizes and the fact that the percentage is comparable to the statistical errors in the ISO sample, our results are formally consistent with those of Lutz et al. (1999) at the $2 \sigma$ level. Our results are more comparable to those of Imanishi et al. (2007), who find that $30 \%-50 \%$ of $\mathrm{H}$ II- and LINER-like ULIRGs contain dominant buried AGNs. Of the 12 LINER-like and six H II-like ULIRGs with low PAH EWs, nine were observed with the high-resolution modules of the IRS (Farrah et al. 2007). Of these, none have detectable [ $\mathrm{Ne} \mathrm{v}] 14.3 \mu \mathrm{m}$, [Ne v] $24.3 \mu \mathrm{m}$, or [O IV] $24.9 \mu \mathrm{m}$ line emission. Upper limits on the [ $\mathrm{Ne} \mathrm{v}] 14.3 \mu \mathrm{m}$ to [ $\mathrm{Ne}$ II $] 12.8 \mu \mathrm{m}$ line flux ratio range from 0.02 to 0.3 , corresponding to starburst-dominated ULIRGs with less

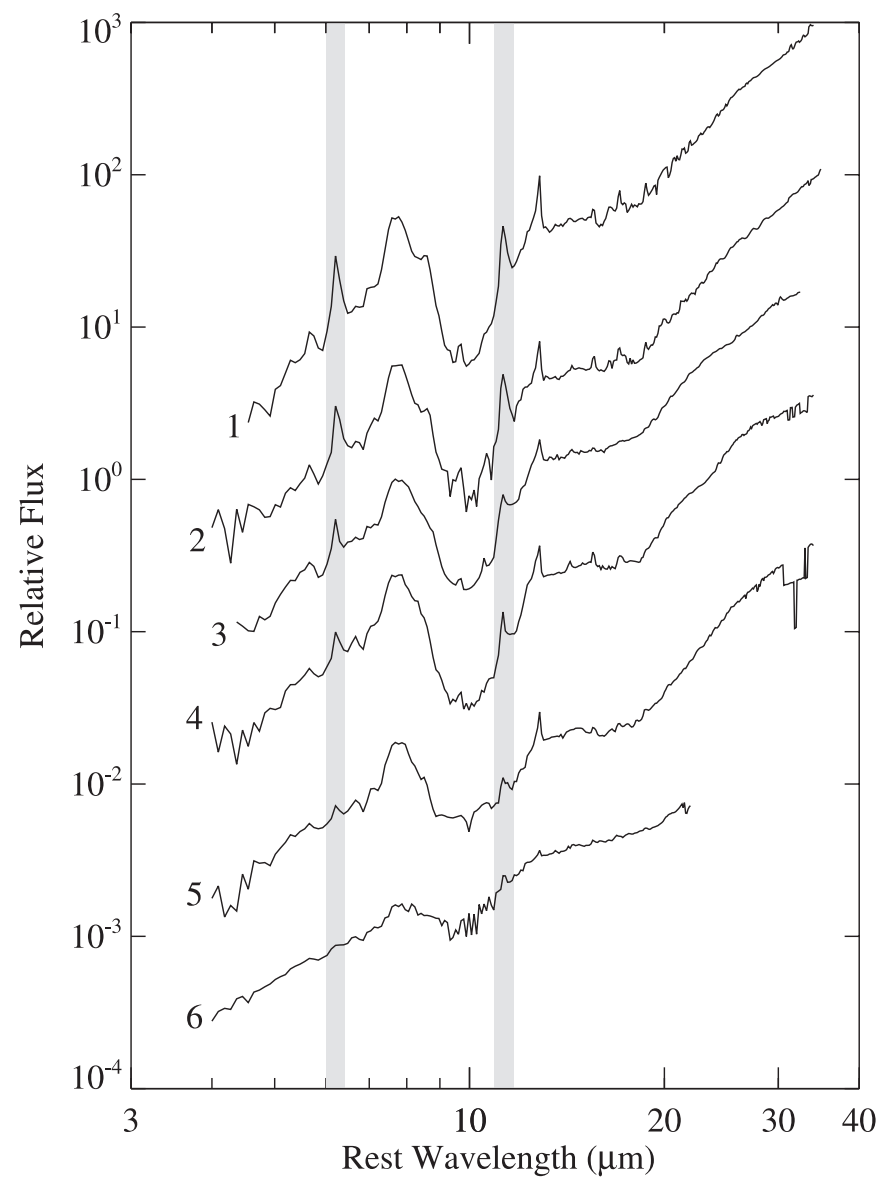

FIG. 6.-Median IRS ULIRG spectra for the six $24 \mu \mathrm{m}$ rest-frame luminosity bins shown in Fig. 5, arranged such that the luminosity increases from top to bottom. The gray bands highlight the positions of the 6.2 and $11.3 \mu \mathrm{m}$ PAH features. than (5-25)\% AGN contribution (Armus et al. 2007). However, it is important to note that many of these sources are deeply buried, as evidenced by their large silicate optical depths (see $\S 3.3$ ). As a result, the $[\mathrm{Ne} \mathrm{v}] 14.3 \mu \mathrm{m}$ emission may be highly extinguished. In addition, it is plausible that a completely buried starburst exists in these sources, as in NGC 1377 (Roussel et al. 2006).

The $6.2 \mu \mathrm{m}$ PAH EW in ULIRGs could also be (intrinsically) smaller than that found in pure starburst galaxies if star formation in ULIRGs is accompanied by larger quantities of hot dust emission than is typically found in lower luminosity starbursts. The dust would then have to "see" more of the stellar radiation and compete more effectively for UV photons, thus raising its average temperature, something that is not unreasonable given the compact nature of the infrared emission in ULIRGs. However, if this were the case, then both the 6.2 and the $11.3 \mu \mathrm{m}$ PAH EWs would be similarly depressed. However, among cold ULIRGs, the median $11.3 \mu \mathrm{m}$ PAH EW is $80 \%$ of that found in starbursts, while the median $6.2 \mu \mathrm{m}$ PAH EW is only $50 \%$. A combination of dilution and extinction offers a more likely explanation. In this scenario, the $6.2 \mu \mathrm{m}$ PAH EW is diluted by hot dust continuum produced by a central ionizing source interior to the star-forming regions. The dilution of the $11.3 \mu \mathrm{m}$ PAH EW is less significant because of its proximity to the broad silicate absorption feature at $9.7 \mu \mathrm{m}$. The hot dust continuum responsible for diluting the $6.2 \mu \mathrm{m} \mathrm{PAH}$ EW is heavily extincted at $11.3 \mu \mathrm{m}$, and therefore does not readily dilute the $11.3 \mu \mathrm{m}$ PAH EW. This is seen in Figure 7. The lefthand panels show that the $6.2 \mu \mathrm{m}$ PAH EWs of ULIRGs on the upper branch deviate strongly from the starbursts. In contrast, the right-hand panels indicate that ULIRGs have nearly starburstlike $11.3 \mu \mathrm{m}$ PAH EWs because the strong extinction at this wavelength overwhelms all but the strongest contribution from hot dust.

Finally, extinction is not likely to play a large role in the reduced $6.2 \mu \mathrm{m}$ PAH EWs found in ULIRGs compared to starbursts. Although ULIRGs on average display stronger silicate absorption features than lower-luminosity starbursts, larger column densities of dust would lead to depressed PAH EWs only if the PAH emission were more extinguished than the continuum. Such a situation is unlikely, since the continuum flux is emitted by hot dust that must lie close to the ionization source.

In a further attempt to disentangle reduced PAH emission from increased hot dust emission as the driving force behind the low $6.2 \mu \mathrm{m}$ PAH EWs in ULIRGs, we plot the $6.2 \mu \mathrm{m}$ PAH luminosity against the rest-frame $5.5 \mu \mathrm{m}$ luminosity in Figure 8. Lowluminosity starbursts from Brandl et al. (2006) follow a correlation between these two quantities, characterized by $\log _{10}[L(6.2 \mu \mathrm{m}$ PAH $)]=-0.49+0.96 \times \log _{10}\left[\nu L_{\nu}(5.5 \mu \mathrm{m})\right]$. ULIRGs do not lie on the extrapolation of this relation to higher luminosities. 


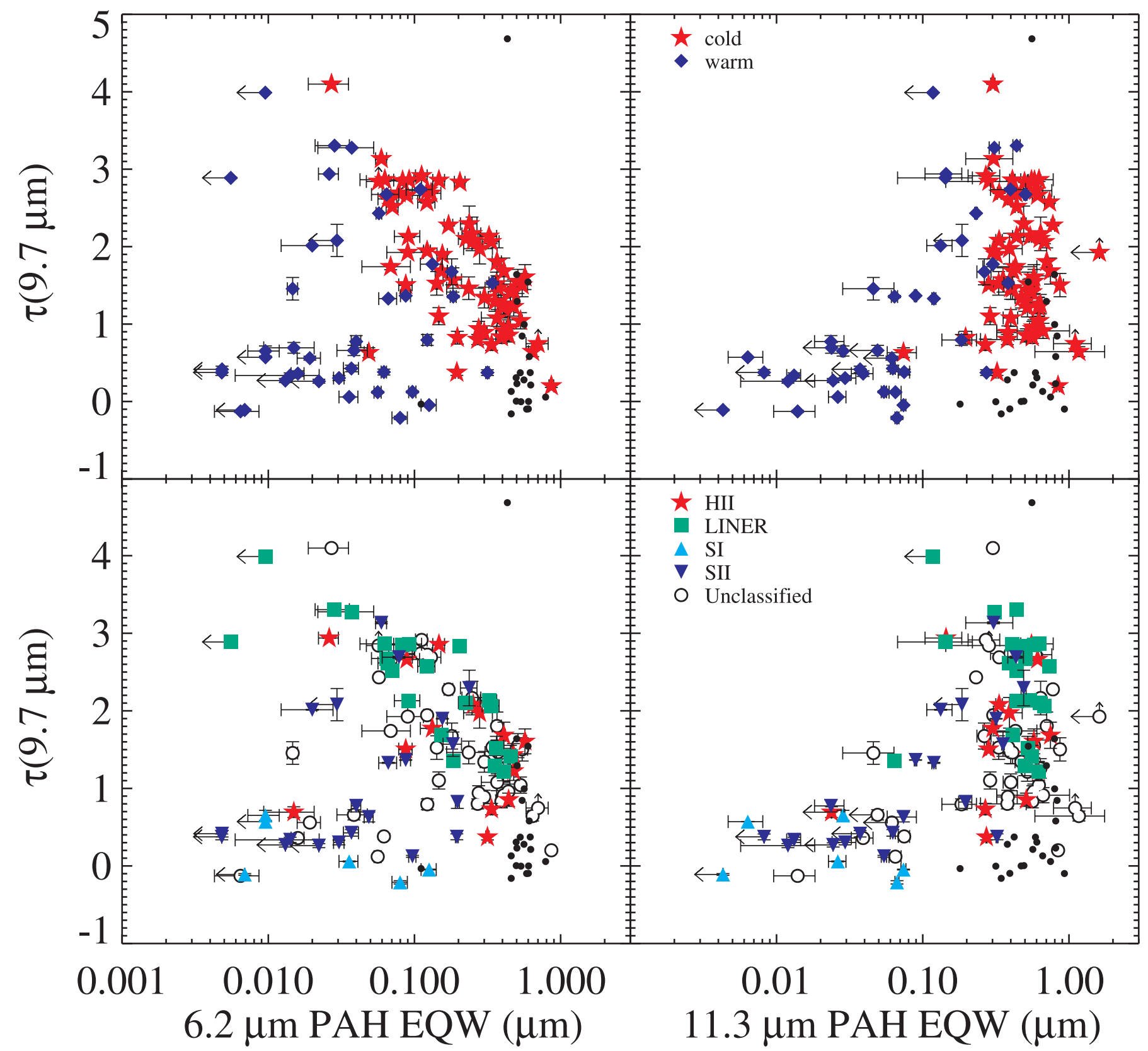

FIG. 7.- Strength of the $9.7 \mu \mathrm{m}$ silicate absorption feature vs. the 6.2 (left $)$ and 11.3 (right) $\mu \mathrm{m}$ PAH EWs. The ULIRGs are color-coded according to spectral slope and optical classification in the left and right panels, respectively. The colored symbols are identical to those in Fig. 5 . The small black dots represent the starbursts from Brandl et al. (2006).

Instead, ULIRGs appear offset to higher $5.5 \mu \mathrm{m}$ luminosities for their measured $6.2 \mu \mathrm{m}$ PAH luminosities. Not surprisingly, sources with flatter far-infrared spectra and those with Seyfert-like optical spectra are, as a group, the most displaced toward higher $5.5 \mu \mathrm{m}$ luminosity. However, the range of $6.2 \mu \mathrm{m}$ PAH luminosity within the different classes (both optical and infrared) is the same. It has been suggested that an evolutionary link exists between ULIRGs and QSOs, such that cold ULIRGs evolve into warm ULIRGs on their way to becoming QSOs (e.g., Sanders et al. 1988a). Figure 8 implies that the transition between cold and warm ULIRGs involves primarily an increase in hot dust, rather than the suppression of PAH emission. Lutz et al. (1998) also suggested that dilution of the EW by hot dust was the dominant effect among ULIRGs.

Also plotted in the lower left-hand panel of Figure 8 are $\sim 80$ Palomar-Green (PG) QSOs at $z<0.5$ (P. Ogle \& R. Antonucci
2007, in preparation; Shi et al. 2007). Most have only upper limits for the $6.2 \mu \mathrm{m}$ PAH EW, but 15 have detected values. The $5.5 \mu \mathrm{m}$ continuum luminosities of ULIRGs and PG QSOs span the same range.

The 15 PG QSOs with detected 6.2 $\mu \mathrm{m}$ PAH features show no evidence for a correlation in Figure 8 . This is in contrast with the results of Schweitzer et al. (2006), who find a correlation between the $7.7 \mu \mathrm{m}$ PAH luminosity and the $6 \mu \mathrm{m}$ continuum luminosity among a sample of 26 PG QSOs, including 15 with upper limits on the PAH luminosity and 11 with detections. Based on the assumptions that the $6 \mu \mathrm{m}$ luminosity is due to hot dust heated by an AGN, while the PAH luminosity is emitted by star-forming regions, they argue that their observed correlation is evidence for a starburstAGN connection among PG QSOs. However, we find no such correlation when considering the $6.2 \mu \mathrm{m}$ PAH feature. 

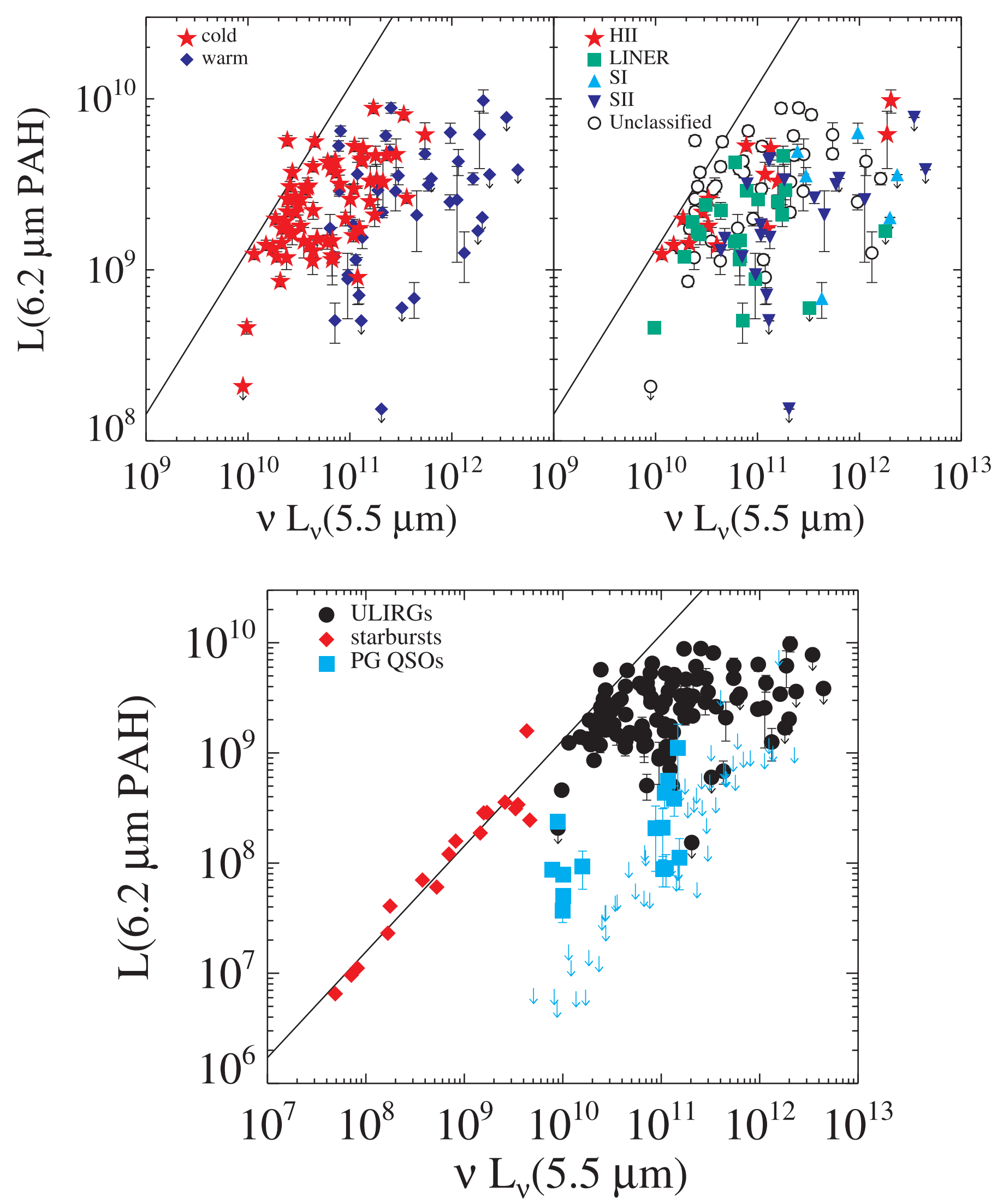

FIG. 8.-Luminosity in the $6.2 \mu \mathrm{m}$ PAH feature as a function of $5.5 \mu \mathrm{m}$ luminosity. In the top panels, the ULIRGs are color-coded according to spectral slope $(l e f t)$ and optical classification (right) using the same symbols as in Fig. 5. The solid black line is a fit to the starbursts analyzed by Brandl et al. (2006). The bottom panel features expanded $x$ - and $y$-axes to show the locations of the Brandl et al. (2006) starbursts (red diamonds) and $\sim 80 z<0.5$ PG QSOs, displayed as cyan squares (detections) and arrows (upper limits). In this bottom panel, the ULIRGs are shown as black circles. 
If an evolutionary link exists between ULIRGs and QSOs, such that cold ULIRGs evolve into warm ULIRGs on their way to becoming QSOs (e.g., Sanders et al. 1988a), we might expect latestage ULIRGs (those with the flattest slopes or Seyfert-like optical spectra) to also show such a correlation. As a class, ULIRGs would not necessarily show a correlation, since the timescale to evolve from starburst to QSO might vary, and we would be catching systems at different stages. However, we might expect ULIRGs which already display optical signatures of an AGN to be near the endpoint in this evolution, and they should display a correlation. It is obvious from Figure 8 that they do not, suggesting that the AGNSB connection, if it exists, must be (re-)established late in the evolution of a ULIRG to a QSO.

While the flatter spectra and lower $6.2 \mu \mathrm{m}$ PAH EWs of many of the ULIRGs can readily be explained by an excess of hot dust emission, the silicate optical depth, at least in some cases, seems inconsistent with a simple model for the emission. The $9.7 \mu \mathrm{m}$ silicate optical depth in ULIRGs varies from $-0.2<\tau_{9.7}<4$, and is on average deeper than in starburst galaxies. The radiative transfer models of Levenson et al. (2007) suggest that the geometry of the obscuring material in ULIRGs determines both the depth of the silicate feature and the infrared spectral slope. According to these models, the large apparent silicate optical depth $\left(\tau_{9.7}>\right.$ 1-2) observed in some ULIRGs requires an optically and geometrically thick, smooth dust component, since a clumpy dust distribution can only result in a shallow $\left(\tau_{9.7}<1\right)$ absorption feature. All of the Seyfert-like ULIRGs would then be viewed through this clumpy dust distribution, exhibiting flat infrared spectra, weak PAH EWs, and shallow (or nonexistent) silicate absorption features. If this simple model is correct, a correlation between silicate optical depth and spectral slope might be expected. However, we find no such correlation (see Fig. 3). In fact, a significant number of the sources with the most extreme absorption are warm (see Fig. 7). These ULIRGs also tend to have low $6.2 \mu \mathrm{m}$ PAH EWs $(<0.15 \mu \mathrm{m})$. A majority of the sources with large silicate optical depth are classified optically as LINERs.

Among local ULIRGs, the sources with the highest rest-frame $24 \mu \mathrm{m}$ luminosities have the lowest $6.2 \mu \mathrm{m}$ PAH EWs (see Fig. 5). The fit to this correlation is shown as the black line in Figure 9, and is given by $\log _{10}[6.2 \mu \mathrm{m} \mathrm{PAH} \mathrm{EW}(\mu \mathrm{m})]=(7.71 \pm 0.07)+$ $(-0.723 \pm 0.006) \times \log _{10}\left(\nu L_{\nu}(24 \mu \mathrm{m})\left[L_{\odot}\right]\right)$. According to this fit, ULIRGs with rest-frame $24 \mu \mathrm{m}$ luminosities above about $10^{12} L$. have $6.2 \mu \mathrm{m}$ PAH EWs below $0.1-0.2 \mu \mathrm{m}$. However, very luminous infrared sources with strong PAH emission $(6.2 \mu \mathrm{m} \mathrm{PAH}$ EW $>0.3-0.5 \mu \mathrm{m}$ ) have recently been found in high-redshift samples that were selected in the submillimeter, which is dominated by cool dust emission (Lutz et al. 2005; Menéndez-Delmestre et al. 2007; Valiante et al. 2007). For example, of the five IRS spectra of submillimeter sources presented by Menéndez-Delmestre et al. (2007), two have the wavelength coverage to measure the $6.2 \mu \mathrm{m}$ PAH feature. These two submillimeter-selected ULIRGs have rest-frame EWs of approximately 0.6 and $0.8 \mu \mathrm{m}$, comparable to the strongest PAH emission measured for local starburst galaxies. Their positions are marked in Figure 9, and they are clearly well above the fit to the local ULIRGs. Additional examples have been found in samples selected at $160 \mu \mathrm{m}$ (MIPS J142824; Desai et al. 2006) or a combination of $24 \mu \mathrm{m}$ flux and mid-infrared color (Yan et al. 2005; Sajina et al. 2007). These are also plotted in Figure 9. However, sources with high rest-frame $24 \mu \mathrm{m}$ luminosities and large $6.2 \mu \mathrm{m}$ PAH EW are rare in bright $\left(f_{24}>0.7 \mathrm{mJy}\right)$ fluxlimited samples selected at $24 \mu \mathrm{m}$ (e.g., Houck et al. 2005; Weedman et al. 2006); these samples appear dominated by AGNs. ULIRGs with high rest-frame $24 \mu \mathrm{m}$ luminosities and strong PAH emission are absent from local samples, yet they obviously exist at

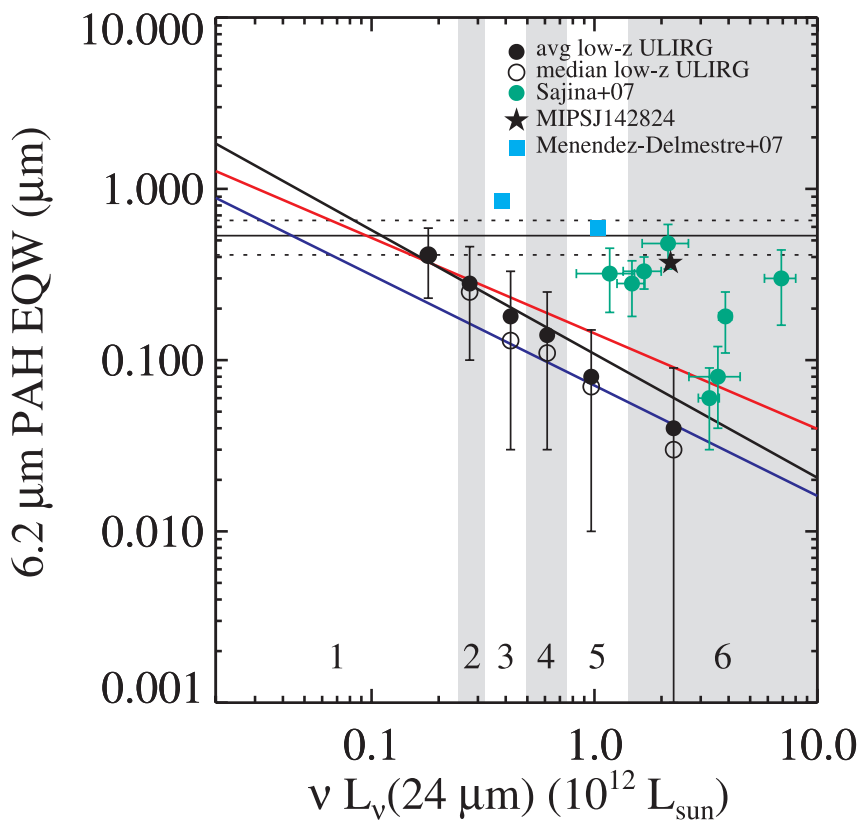

FIG. 9.-Median (open circles) and average ( filled circles) $6.2 \mu \mathrm{m}$ PAH EWs vs. $24 \mu \mathrm{m}$ luminosity. Both the medians and the means were computed by including sources with PAH EW upper limits in the same way as detections. The error bars were computed as the dispersion in the PAH EWs in each luminosity bin, and are centered on the average values in this plot. The $x$-position of each point is the median of the $24 \mu \mathrm{m}$ luminosities of the sources in each luminosity bin. The thick red, blue, and black lines are fits to the cold ULIRGs, the warm ULIRGs, and all of the ULIRGs, respectively. Sources with upper limits on the PAH EW were included in the fits as detections with $10 \%$ error bars. The horizontal thin and dotted black lines have the same meaning as in Fig. 5 , as do the luminosity bin labels across the bottom. The green points are the high-redshift $(z \sim 2)$ ULIRGs from Sajina et al. (2007) with measured $6.2 \mu \mathrm{m}$ PAH EWs, and rest-frame $24 \mu \mathrm{m}$ luminosities estimated from the $70 \mu \mathrm{m}$ MIPS photometry. The large black star is MIPS J142824.0+ 352619 , a bright $160 \mu \mathrm{m}$ source at $z=1.3$. The rest-frame $24 \mu$ m luminosity for this object was computed by via log-log interpolation of the photometry presented in Borys et al. (2006) and the 6.2 $\mu \mathrm{m}$ PAH EW was taken from Desai et al. (2006). The blue squares are the submillimeter galaxies with measured 6.2 $\mu \mathrm{m}$ PAH EWs from Menéndez-Delmestre et al. (2007). Their rest-frame $22 \mu \mathrm{m}$ luminosities were estimated via SED fitting of the observed photometry at $24 \mu \mathrm{m}, 350 \mu \mathrm{m}$, $850 \mu \mathrm{m}$, and 1.4 GHz (Menéndez-Delmestre et al. 2007; Kóvacs et al. 2006; Chapman et al. 2005).

high redshift, and they can be found by selecting on color or cold dust emission, or by probing to fainter flux levels in the midinfrared (observed $f_{24}<0.3 \mathrm{mJy}$ ). At $z<1$, the generation of large amounts of warm dust seems to require the presence of a dominant AGN. The absence of extremely luminous starburstdominated ULIRGs at low redshift represents a real effect, and not simply an observational bias.

\section{SUMMARY AND CONCLUSIONS}

Using a large sample of 107 ULIRGs observed with the IRS on board the Spitzer Space Telescope, we investigate the relationships between the 6.2 and $11.3 \mu \mathrm{m}$ PAH EWs, continuum luminosity, infrared spectral slope, optical spectroscopic classification, and silicate optical depth. Our results can be summarized as follows:

1. There is an extremely large range in detected $6.2 \mu \mathrm{m} \mathrm{PAH}$ EWs among ULIRGs, spanning more than 2 orders of magnitude from $\sim 0.006$ to $0.9 \mu \mathrm{m}$. ULIRGs classified as starburst-dominated based on their far-infrared colors or optical spectra have median $6.2 \mu \mathrm{m}$ PAH EWs that are $50 \%$ of that measured in low-luminosity starburst galaxies. An excess of hot dust, which is most prominent in the far-infrared warm or Seyfert-like ULIRGs, appears to be the 
cause of the decreased PAH EW, as opposed to extinction or grain destruction. Approximately 55\% of LINER-like ULIRGs, $40 \%$ of H II-like ULIRGs, and 35\% of cold ULIRGs have very low $6.2 \mu \mathrm{m}$ PAH EWs, indicative of buried AGN or starbursts with suppressed PAH emission. Most of these ULIRGs also have high apparent silicate optical depths at $9.7 \mu \mathrm{m}$.

2. The apparent silicate optical depth is not correlated with far-infrared spectral slope. The ULIRGs with the strongest silicate absorption features have small $6.2 \mu \mathrm{m}$ PAH EWs and flat spectral slopes in the far-infrared. Their strong silicate absorption implies large columns of cold dust, but their small PAH EWs and farinfrared colors imply substantial amounts of hot dust emission. For these sources, a model in which a warm source is viewed through either a smooth optically thick or clumpy dust shell seems inconsistent with the data.
3. ULIRGs with the largest rest-frame $24 \mu \mathrm{m}$ luminosities have the smallest $6.2 \mu \mathrm{m}$ PAH EWs. A fit to our sample suggests $\log _{10}(6.2 \mu \mathrm{m}$ PAH EW $[\mu \mathrm{m}])=(7.71 \pm 0.07)+(-0.723 \pm$ $0.006) \times \log _{10}\left(\nu L_{\nu}(24 \mu \mathrm{m})\left[L_{\odot}\right]\right)$ The range in PAH EWs among luminous, high-redshift sources observed with Spitzer is much larger than implied by this correlation due to the presence of luminous sources with very large $6.2 \mu \mathrm{m}$ PAH EWs. Extremely luminous sources of all types are rare at low-redshift, but those that do exist seem to be AGN-dominated.

We would like to thank R. Chary, M. Lacy, J. Surace, and A. Sajina for insightful discussions. Support for this work was provided by NASA through an award issued by JPL/Caltech.
Aitken, D. K., \& Roche, P. F. 1985, MNRAS, 213, 777

Allen, D. A., Norris, R. P., Meadows, V. S., \& Roche, P. F. 1991, MNRAS, 248, 528

Armus, L., Heckman, T., \& Miley, G. 1987, AJ, 94, 831 1989, ApJ, 347, 727

Armus, L., et al. 2004, ApJS, 154, 178

2006, ApJ, 640, 204

2007, ApJ, 656, 148

Borys, C., et al. 2006, ApJ, 636, 134

Brandl, B. R., et al. 2006, ApJ, 653, 1129

Burston, A. J., Ward, M. J., \& Davies, R. I. 2001, MNRAS, 326, 403

Chapman, S. C., Blain, A. W., Smail, I., \& Ivison, R. J. 2005, ApJ, 622, 772

Cutri, R. M., Huchra, J. P., Low, F. J., Brown, R. L., \& Vanden Bout, P. A. 1994, ApJ, 424, L65

Dannerbauer, H., Rigopoulou, D., Lutz, D., Genzel, R., Sturm, E., \& Moorwood, A. F. M. 2005, A\&A, 441, 999

Davies, R. I., Sternberg, A., Lehnert, M., \& Tacconi-Garman, L. E. 2003, ApJ, 597, 907

de Grijp, M. H. K., Miley, G. K., Lub, J., \& de Jong, T. 1985, Nature, 314, 240

Desai, V., et al. 2006, ApJ, 641, 133

Duc, P.-A., Mirabel, I. F., \& Maza, J. 1997, A\&AS, 124, 533

Farrah, D., et al. 2007, 667, 149

Franceschini, A., Aussel, H., Cesarsky, C. J., Elbaz, D., \& Fadda, D. 2001, A\&A, 378, 1

Genzel, R., et al. 1998, ApJ, 498, 579

Goldader, J. D., Joseph, R. D., Doyon, R., \& Sanders, D. B. 1995, ApJ, 444, 97

Hao, L., Weedman, D. W., Spoon, H. W. W., Marshall, J. A., Levenson, N. A., Elitzur, M., \& Houck, J. R. 2007, ApJ, 655, L77

Higdon, S. J. U., et al. 2004, PASP, 116, 975

Houck, J. R., et al. 2004, ApJS, 154, 18

2005, ApJ, 622, L105

Imanishi, M., Dudley, C. C., Maiolino, R., Maloney, P. R., Nakagawa, T., \& Risaliti, G. 2007, ApJS, 171, 72

Kim, D.-C., Veilleux, S., \& Sanders, D. B. 1998, ApJ, 508, 627

Kleinmann, S. G., Hamilton, D., Keel, W. C., Wynn-Williams, C. G., Eales, S. A., Becklin, E. E., \& Kuntz, K. D. 1988, ApJ, 328, 161

Kormendy, J., \& Sanders, D. B. 1992, ApJ, 390, L53

Kóvacs, A., Chapman, S. C., Dowell, C. D., Blain, A. W., Ivison, R. J., Smail, I., \& Phillips, T. G. 2006, ApJ, 650, 592

Laurent, O., Mirabel, I. F., Charmandaris, V., Gallais, P., Madden, S. C., Sauvage, M., Vigroux, L., \& Cesarsky, C. 2000, A\&A, 359, 887

Le Floc'h, E., et al. 2005, ApJ, 632, 169

Levenson, N. A., Sirocky, M. M., Hao, L., Spoon, H. W. W., Marshall, J. A., Elitzur, M., \& Houck, J. R. 2007, ApJ, 654, L45

Lutz, D., Spoon, H. W. W., Rigopoulou, D., Moorwood, A. F. M., \& Genzel, R. 1998, ApJ, 505, L103

Lutz, D., Valiante, E., Sturm, E., Genzel, R., Tacconi, L. J., Lehnert, M. D., Sternberg, A., \& Baker, A. J. 2005, ApJ, 625, L83

Lutz, D., Veilleux, S., \& Genzel, R. 1999, ApJ, 517, L13

\section{EFERENCES}

Menéndez-Delmestre, K., et al. 2007, ApJ, 655, L65

Moshir, M. 1990, in IRAS Faint Source Catalogue, version 2.0

Murphy, T. W., Jr., Soifer, B. T., Matthews, K., Armus, L., \& Kiger, J. R. 2001, AJ, 121, 97

Murphy, T. W., Jr., Soifer, B. T., Matthews, K., Kiger, J. R., \& Armus, L. 1999, ApJ, 525, L85

Osterbrock, D. E., \& De Robertis, M. M. 1985, PASP, 97, 1129

Pérez-González, P. G., et al. 2005, ApJ, 630, 82

Rigopoulou, D., Spoon, H. W. W., Genzel, R., Lutz, D., Moorwood, A. F. M., \& Tran, Q. D. 1999, AJ, 118, 2625

Roussel, H., et al. 2006, ApJ, 646, 841

Sajina, A., Yan, L., Armus, L., Choi, P., Fadda, D., Helou, G., \& Spoon, H. 2007, 667, L17

Sanders, D. B., Mazzarella, J. M., Kim, D.-C., Surace, J. A., \& Soifer, B. T. 2003, AJ, 126, 1607

Sanders, D. B., \& Mirabel, I. F. 1996, ARA\&A, 34, 749

Sanders, D. B., Soifer, B. T., Elias, J. H., Madore, B. F., Matthews, K., Neugebauer, G., \& Scoville, N. Z. 1988a, ApJ, 325, 74

Sanders, D. B., Soifer, B. T., Elias, J. H., Neugebauer, G., \& Matthews, K. 1988b, ApJ, 328, L35

Schweitzer, M., et al. 2006, ApJ, 649, 79

Scott, S. E., et al. 2002, MNRAS, 331, 817

Shi, Y., et al. 2007, ApJ, submitted (arXiv: 0707.2806)

Soifer, B. T., Sanders, D. B., Madore, B. F., Neugebauer, G., Danielson, G. E., Elias, J. H., Lonsdale, C. J., \& Rice, W. L. 1987, ApJ, 320, 238

Soifer, B. T., et al. 2000, AJ, 119, 509

Spoon, H. W. W., Keane, J. V., Tielens, A. G. G. M., Lutz, D., Moorwood, A. F. M., \& Laurent, O. 2002, A\&A, 385, 1022

Spoon, H. W. W., Marshall, J. A., Houck, J. R., Elitzur, M., Hao, L., Armus, L., Brandl, B. R., \& Charmandaris, V. 2007, ApJ, 654, L49

Stanford, S. A., Stern, D., van Breugel, W., \& De Breuck, C. 2000, ApJS, 131, 185

Strauss, M. A., Huchra, J. P., Davis, M., Yahil, A., Fisher, K. B., \& Tonry, J. 1992, ApJS, 83, 29

Sturm, E., Lutz, D., Verma, A., Netzer, H., Sternberg, A., Moorwood, A. F. M., Oliva, E., \& Genzel, R. 2002, A\&A, 393, 821

Sturm, E., et al. 2006, ApJ, 653, L13

Tran, Q. D., et al. 2001, ApJ, 552, 527

Valiante, E., Lutz, D., Sturm, E., Genzel, R., Tacconi, L. J., Lehnert, M. D., \& Baker, A. J. 2007, ApJ, 660, 1060

Veilleux, S., Kim, D.-C., \& Sanders, D. B. 1999a, ApJ, 522, 113

Veilleux, S., Kim, D.-C., Sanders, D. B., Mazzarella, J. M., \& Soifer, B. T. 1995, ApJS, 98, 171

Veilleux, S., Sanders, D. B., \& Kim, D.-C. 1997, ApJ, 484, 92 1999b, ApJ, 522, 139

Voit, G. M. 1992, MNRAS, 258, 841

Weedman, D. W., et al. 2006, ApJ, 651, 101

Yan, L., et al. 2005, ApJ, 628, 604 